\title{
Anisotropically oriented electrospun matrices with an imprinted periodic micropattern: a new scaffold for engineered muscle constructs
}

\author{
Anne Géraldine Guex ${ }^{1,2}$, Dominique Lisa Birrer ${ }^{2}$, \\ Giuseppino Fortunato $^{1,4}$, Hendrik Tinorua Tevaearai ${ }^{2}$ \\ and Marie-Noëlle Giraud ${ }^{3}$ \\ ${ }^{1}$ Empa-Swiss Federal Laboratories for Materials Science and Technology, Lerchenfeldstrasse 5, \\ 9014 St Gallen, Switzerland \\ ${ }^{2}$ Clinic for Cardiovascular Surgery, Inselspital Bern, University Hospital and University of Bern, \\ Murtenstrasse 35, 3010 Bern, Switzerland \\ ${ }^{3}$ Cardiology, Department of Medicine, Faculty of Science, University of Fribourg, Chemin du Musée 5, \\ 1700 Fribourg, Switzerland \\ E-mail: geraldine.guex@empa.ch, dominique.birrer@students.unibe.ch, marie-noelle.giraud@unifr.ch, \\ giuseppino.fortunato@empa.ch and hendrik.tevaearai@insel.ch
}

\begin{abstract}
Engineered muscle constructs provide a promising perspective on the regeneration or substitution of irreversibly damaged skeletal muscle. However, the highly ordered structure of native muscle tissue necessitates special consideration during scaffold development. Multiple approaches to the design of anisotropically structured substrates with grooved micropatterns or parallel-aligned fibres have previously been undertaken. In this study we report the guidance effect of a scaffold that combines both approaches, oriented fibres and a grooved topography. By electrospinning onto a topographically structured collector, matrices of parallel-oriented $\operatorname{poly}(\varepsilon$-caprolactone) fibres with an imprinted wavy topography of $90 \mu$ m periodicity were produced. Matrices of randomly oriented fibres or parallel-oriented fibres without micropatterns served as controls. As previously shown, un-patterned, parallel-oriented substrates induced myotube orientation that is parallel to fibre direction. Interestingly, pattern addition induced an orientation of myotubes at an angle of $24^{\circ}$ (statistical median) relative to fibre orientation. Myotube length was significantly increased on aligned micropatterned substrates in comparison to that on aligned substrates without pattern (436 $\pm 245 \mu \mathrm{m}$ versus $365 \pm 212 \mu \mathrm{m} ; p<0.05)$. We report an innovative, yet simple, design to produce micropatterned electrospun scaffolds that induce an unexpected myotube orientation and an increase in myotube length.
\end{abstract}




\section{Introduction}

In the last decade, biomaterial sciences have broadly contributed to the emergence of new therapeutic approaches in regenerative muscle medicine. Engineered muscle constructs, composed of an adequate scaffold and autologous muscle cells represent a promising treatment for patients with either irreversible skeletal muscle damage or insufficient intrinsic muscle repair capacity (Koning et al 2009, Rossi et al 2010). The design of a tissue-specific scaffold, however, faces numerous challenges related to material choice and processing. Indeed, the influence of mechanical, chemical and architectural properties of the substrate material on the morphology, proliferation and differentiation of myoblasts has been clearly demonstrated (Engler et al 2004, Riboldi et al 2005, Choi et al 2008, Guex et al 2012, Ricotti et al 2012).

During native myogenesis, generation of a primary myotube layer with a highly oriented pattern provides a substrate for further myotube incorporation, comprising a strongly hierarchical mechanism to direct muscle tissue formation (Ontell 1977, Duxson et al 1989, Hauschka et al 1994, Zhang and McLennan 1995, Wigmore and Dunglison 1998). Secondary myotubes come to lie within micron-scaled grooves generated by the primary myotube layer to build-up the tissue. Hence, it is now widely accepted that inducing myoblast alignment prior to myotube fusion (Wakelam 1985), and controlling the in vitro tissue organization, is of particular importance for functional muscle tissue engineering. Accordingly, integrated modulations of scaffold surface architecture at the nano- or micron-scale with parallel-oriented structures (often referred to anisotropic scaffolds) have been used to promote spatial alignment of myoblasts. Among different approaches, patterned polydimethylsiloxane (PDMS) substrates were designed using lithography of UV light, electron beam or ion beam. Micron-scale periodic grooves have been shown to increase myoblast orientation (Lam et al 2006, Charest et al 2007, Gingras et al 2009, Shimizu et al 2009, Altomare et al 2010). Myoblasts and myotubes aligned on substrates with groove widths of $0.45-50 \mu \mathrm{m}$, with a minimal threshold requirement of $130 \mathrm{~nm}$. An alternative strategy consists of electrospinning synthetic polymers to produce parallel-oriented nano- or micron-scaled fibres (Riboldi et al 2005, Choi et al 2008, Aviss et al 2010, Ricotti et al 2012). Additionally, we have recently shown (Guex et al 2012) that myoblasts align preferentially on parallel-oriented nano- to submicron-sized fibres of poly $(\varepsilon$ caprolactone) (PCL), as compared to those randomly oriented.

We investigated the hypothesis that the dual-level, fibrous topography of periodic grooves closely mimics the structure of the primary myotube layer, formed during foetal myogenesis (Ontell 1977, Duxson et al 1989, Hauschka et al 1994), thereby proving an adequate scaffold for myotube formation. We aimed for the design of a topography without sharp boundaries that supports lateral, as well as longitudinal migration and fusion of myoblasts, generating wider and longer myotubes. To do so, we developed an innovative, yet very simple, method to produce micropatterned matrices of parallel-oriented fibres. Despite tremendous effort on both micropatterned substrates
Table 1. Electrospinning process parameters applied to produce fibrous matrices.

\begin{tabular}{lllll}
\hline Matrix & Collector & $\begin{array}{l}\text { Distance } \\
(\mathrm{cm})\end{array}$ & $\begin{array}{l}\text { Voltage } \\
(\mathrm{kV})\end{array}$ & $\begin{array}{l}\text { Flow rate } \\
\left(\mu \mathrm{L} \mathrm{min} \mathrm{m}^{-1}\right)\end{array}$ \\
\hline $\begin{array}{l}\text { Randomly- } \\
\text { oriented }\end{array}$ & Static (plate) & 15 & $10 /-2$ & 7 \\
$\begin{array}{l}\text { Parallel- } \\
\text { aligned }\end{array}$ & Rotating drum & 15 & $10 /-5$ & $7-10$ \\
$\begin{array}{l}\text { Micropatterned } \\
\text { Rotating drum } \\
\text { with relief }\end{array}$ & 20 & $10 /-4$ & 10 \\
\hline
\end{tabular}

and electrospun matrices, these two approaches have rarely been combined. These matrices were evaluated using a cell culture of murine myoblasts ( $\mathrm{C} 2 \mathrm{C} 12$ cell line). Cell viability, proliferation, orientation and differentiation were assessed in response to surface architecture.

\section{Materials and methods}

\subsection{Substrate production and characterization}

Poly( $\varepsilon$-caprolactone) (PCL, $\left.80000 \mathrm{~g} \mathrm{~mol}^{-1}\right)$, glacial acetic acid $\left(\mathrm{CH}_{3} \mathrm{COOH}, 99 \%\right)$ and pyridine $\left(\mathrm{C}_{5} \mathrm{H}_{5} \mathrm{~N}, \geqslant 99 \%\right)$, were purchased from Sigma-Aldrich, Buchs, Switzerland. PCL was dissolved in glacial acetic acid/pyridine (100:1) to obtain a $15 \% \mathrm{w} / \mathrm{v}$ solution. Fibrous matrices were produced by electrospinning as previously reported (Moghe et al 2009, Guex et al 2012). Briefly, the apparatus consisted of a positive (applied to the needle) and a negative (applied to the collector) voltage supply source (aip Wild AG, Oberglatt, Switzerland) that maintained an electrical field. A constant polymer flow of the spinning solution was provided by an infusion pump (KD Scientific, Holliston, USA). Spinning parameters are displayed in table 1. Randomly-oriented fibres were assembled on a planar steel plate. Anisotropic scaffolds of parallel-oriented fibres were collected on a rotating drum (about $1000 \mathrm{rpm}$ ), whereas parallel-oriented fibres with an additional periodic pattern were produced by spinning onto a rotating drum with an engraved relief. The relief consisted of a continuous wavy pattern of $90 \mu \mathrm{m}$ periodicity at a depth of $14 \mu \mathrm{m}$ (figure 1). The relief was engraved during the production of the drum.

Matrix architecture and fibre diameter were characterized by scanning electron microscopy (SEM, Hitachi S-4800; Hitachi High-Technologies US and Canada, Illinois, USA). Substrates were plasma gold sputtered (Polaron Equipment, SEM coating Unit E5100, Kontron AG, Switzerland; 5 mA, 1 mbar) prior to image acquisition at an accelerating voltage of $2 \mathrm{kV}$ and $10 \mu \mathrm{A}$ current flow. For fibre diameter evaluation, 100 values per condition were measured.

Mechanical properties were assessed on an Instron 4500 apparatus (Instron, Norwood, USA) as previously reported (Guex et al 2012). Substrates of $600 \mathrm{~mm}^{2}$ were conditioned overnight at $25^{\circ} \mathrm{C}$ and $65 \%$ relative humidity and stress-strain curves were recorded at a displacement rate of $25 \mathrm{~mm} \mathrm{~min}^{-1}$. Stress was normalized to the cross sectional surface area, taking into account the cross sectional area of the patch. Three measurements per matrix type were performed. 

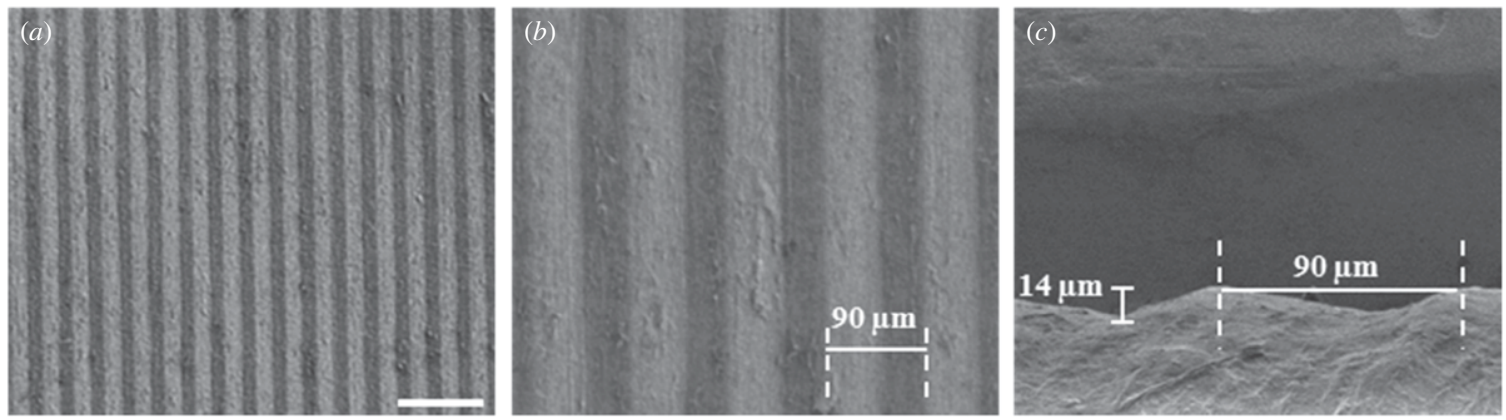

Figure 1. SEM images of the relief on the drum. A cast PCL film was used to visualize engraved topography on the drum $(a)$ and $(b)$ top view, (c) cross sectional view. The pattern had a periodicity of $90 \mu \mathrm{m}$ and a depth of $14 \mu \mathrm{m}$. Scale bar on panel $(a)$ represents $300 \mu \mathrm{m}$.

\subsection{Cell culture}

All experiments were carried out with mouse skeletal myoblasts, cell line C2C12 (ATCC, Manassas, USA) under aseptic conditions using sterile, RNAse/DNAse-free tissue culture plastic (BD, Biosciences, San José, USA and TCPS, TPP Omnilab, Mettmenstetten, Switzerland). Cell culture was maintained at $37{ }^{\circ} \mathrm{C}$ and $5 \% \mathrm{CO}_{2}$ in a humidified incubator (Thermo Forma, Model 371, Thermo Fisher Scientific, Waltham, USA). Experiments were performed in triplicate.

Expansion of $\mathrm{C} 2 \mathrm{C} 12$ cells was carried out in growth medium (Ham F-12, Gibco, Invitrogen, Carlsbad, USA) enriched with $10 \%$ foetal bovine serum (FBS, PAA clone, Connectorate AG, Dietikon, Switzerland), $1.25 \%$ penicillin/streptomycin solution $\left(\mathrm{P} / \mathrm{S}, 100 \mu \mathrm{g} \mathrm{mL}^{-1}\right.$, Gibco, Invitrogen, Carlsbad, USA) and supplemented with $5 \mathrm{ng}$ $\mathrm{mL}^{-1}$ human fibroblast growth factor-basic (bFGF, SigmaAldrich, Buchs, Switzerland) and $40 \mu \mathrm{g} \mathrm{mL}^{-1}$ dexamethasone (Dex, Sigma-Aldrich, Buchs, Switzerland). Myotube differentiation was induced by changing from growth medium to serum deficient differentiation medium (Dulbecco's Modified Eagle Medium (DMEM, Gibco, Invitrogen, Carlsbad, USA), supplemented with $7 \%$ temperature decomplemented horse serum (Gibco, Invitrogen, Carlsbad, USA) and $100 \mu \mathrm{g} \mathrm{mL}^{-1} \mathrm{P} / \mathrm{S}$ ). Medium was refreshed every other day.

For cell culture on matrices, culture dishes (Costar, Corning Incorporated, Corning, USA) were coated with silicone (Sylgard-184 two component silicone, Dow Corning Corporation, Midland, USA). Blank matrices of $6 \mathrm{~mm}$ diameter were fixed on the silicone coatings with stainless steel minutiae insect pins (EntoSPHINX, Pardubice, Czech Republic). Matrices were sterilized under UV-light overnight. Cell seeding was accomplished by deposing $0.25 \times 10^{6}$ cells in $50 \mu \mathrm{L}$ medium on the matrices. Cells were cultured for three days in growth medium, and further maintained in differentiation medium for ten days.

2.2.1. Cell viability and proliferation. An MTT assay (3(4,5-dimethylthiazol-2-yl)-2,5-diphenyltetrazolium bromide, Sigma-Aldrich, Buchs, Switzerland) was used for cell viability assessment. Spectrophotometrical quantification (Infinite 200, Tecan Trading AG, Männedorf, Switzerland at $540 \mathrm{~nm}$ maximal absorption) was performed following $2 \mathrm{~h}$ incubation with $0.5 \mathrm{mg} \mathrm{mL}{ }^{-1}$ MTT in culture medium, followed by transfer to $100 \%$ isopropanol (Spitalpharmazie, Inselspital, Bern, Switzerland).

Substrate toxicity, quantified by lactate dehydrogenase (LDH) release into culture medium was assessed on days 3, 5 and 7 using an LDH kit (Tox-7, Sigma-Aldrich, Buchs, Switzerland). During medium changes, supernatant was collected, incubated with the provided solutions and quantified spectrophotometrically at $490 \mathrm{~nm}$ absorbance (Infinite 200, Tecan Trading AG, Männedorf, Switzerland). LDH release was compared to total LDH release of lysed cells.

To determine the time of highest cell confluence for initiation of differentiation on the substrates, cell number was assessed by DNA quantification on days 3, 5 and 7 (CyQuant, cell proliferation assay, Invitrogen, Carlsbad, USA). Constructs were incubated in cell lysis buffer, sonicated (Sonopuls HD 2070/UW 2070, Bandelin Electronic, Berlin, Germany), vortexed, centrifuged and incubated with a DNA binding agent. Quantification was accomplished spectrophotometrically (Infinite 200, Tecan Trading AG, Männedorf, Switzerland, at $480 \mathrm{~nm}$ excitation and $520 \mathrm{~nm}$ emission). For cell viability and proliferation analysis, $n=12$.

\subsubsection{Myoblast and myotube orientation. Cell angle} analysis, (NISelements BR software, Nikon, Melville, USA) was used to estimate spatial cell orientation with respect to fibre alignment. Myoblasts were stained for desmin (antidesmin antibody, Abd Serotec, USA; 1:200) in phosphate buffered saline (PBS, Spitalpharmazie, Inselspital, Bern, Switzerland) by means of a commercially available staining kit (EnVision System-HRP Kit, Dako, USA). Briefly, substrates were fixed in 4\% formaldehyde (Institute of Pathology, Inselspital, Bern, Switzerland), permeabilized with $0.2 \%$ triton X-100 (Sigma-Aldrich, Buchs, Switzerland), blocked with peroxidase complex, and labelled with antimouse streptavidine-HRP. 3-amino-9-ethylcarbazole was added and enzymatically converted to a red chromophore. Constructs were counterstained with haematoxylin (Mayer's haematoxylin solution, Sigma-Aldrich, Buchs, Switzerland) and mounted with Glycergel (Dako Schweiz AG, Baar, Switzerland).

Cell orientation was quantified using the following orientation parameter:

$$
S=\frac{1}{n} \sum_{i=1}^{n} \cos \left(2 \alpha_{i}\right),
$$



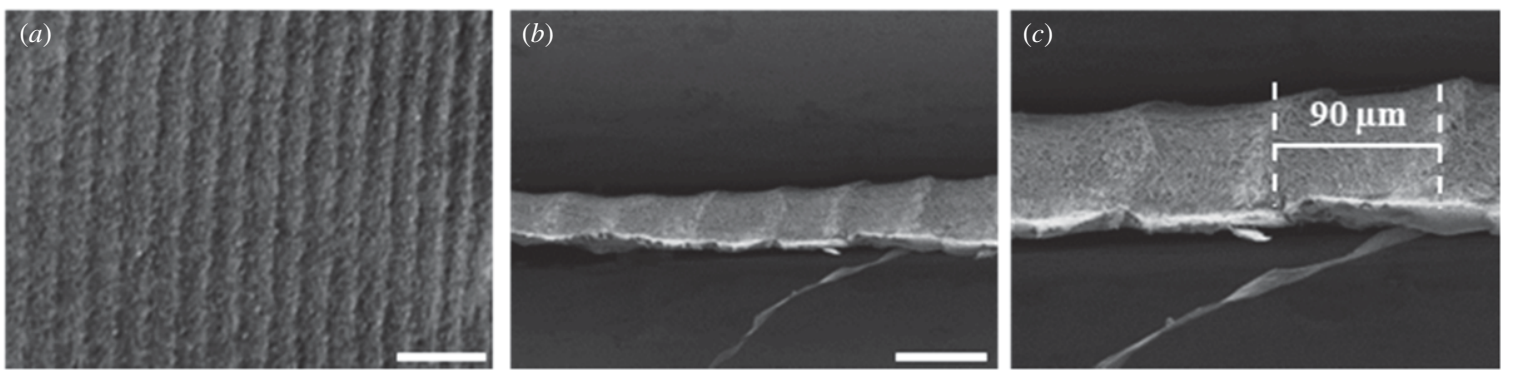

Figure 2. SEM images of the micropatterned nanofibrous substrate. The relief has been copied to the fibrous scaffold, the periodic pattern is clearly visible. Scale bars represent $300 \mu \mathrm{m}(a)$ and $100 \mu \mathrm{m}(b)$.

where $n=$ number of cells, $\alpha=$ cell angle, $S=1$ for aligned cells and $S=0$ for randomly oriented cells (Erickson and Nuccitelli 1984, Biela et al 2009). 1800 cell angles per condition were assessed. Myotube orientation was similarly evaluated $(n=450)$.

\subsubsection{Myotube differentiation. ImageJ (free download at http://rsbweb.nih.gov/ij/download.html) was employed to assess length, width and cross sectional area of desmin $^{(+)}$stained myotubes. The degree of differentiation is expressed as percentage of myotube coverage, whereas myotube width and length are expressed as mean value $\pm \mathrm{SD}$, assessed on 300 individual myotubes per condition.}

\subsection{Statistical analysis}

All values are expressed as mean \pm SD. Data were analysed using SPSS for Windows (version 17.0; SPSS Inc., Chicago, USA). Statistical analysis was performed with an ANOVA, followed by pair-wise comparisons between groups, using unpaired $t$-tests with Bonferroni adjustments. Two-sided corrected $P$-values were considered statistically significant for $p \leqslant 0.05$.

A power analysis was accomplished using the free Java Applets for power and sample size, retrieved from http://www.stat.uiowa.edu/ rlenth/Power (Lenth 2006-2009). We calculated the sample size required to achieve a power of 0.95 given that $\alpha=0.05$. Furthermore, a retrospective calculation of the statistical power for a two sampled $t$-test was performed.

\section{Results and discussion}

\subsection{Substrate characterization}

In this study, two approaches were combined to produce micropatterned, anisotropic electrospun matrices. Different collectors allowed for the production of PCL matrices displaying distinct fibre orientation and surface topography: spinning onto a static steel plate resulted in matrices displaying randomly-oriented nano scaled fibres (n-RO); whereas spinning onto a drum, rotating at a speed of about $1000 \mathrm{rpm}$, resulted in the generation of parallel-aligned nanofibres (n-AL). Additionally, we used an original approach to produce electrospun fibres with a periodic pattern and a distinct surface topography (n-TOP). The surface structure was
Table 2. Mechanical properties of investigated matrices.

\begin{tabular}{llccc}
\hline & $\begin{array}{c}\text { Fibre } \\
\text { Matrix }\end{array}$ & $\begin{array}{c}\text { Elastic } \\
\text { diameter }(\mathrm{nm})\end{array}$ & $\begin{array}{c}\text { Ultimate } \\
\text { modulus }(\mathrm{MPa})\end{array}$ & $\begin{array}{c}\text { Elongation } \\
\text { strength }(\mathrm{MPa})\end{array}$ \\
\hline $\mathrm{at}$ break (\%) \\
$\mathrm{n}$-RO & $265 \pm 97$ & $7 \pm 4$ & $4 \pm 3$ & $161 \pm 56$ \\
$\mathrm{n}-\mathrm{AL}$ & $354 \pm 156$ & $17 \pm 8$ & $14 \pm 3$ & $64 \pm 15$ \\
$\mathrm{n}-\mathrm{TOP}$ & $148 \pm 43^{\mathrm{a}}$ & $36 \pm 16$ & $15 \pm 4$ & $44 \pm 7$ \\
\hline
\end{tabular}

${ }^{a} p<0.01$ versus $\mathrm{n}-\mathrm{AL}$ and $\mathrm{n}-\mathrm{RO}(n=100$, power $\pi=1)$.

transferred to the fibrous matrix, resulting in a wavy structure of grooves and ridges with $90 \mu \mathrm{m}$ periodicity (figure 2). We suggest that the latter fibres first filled the grooves of the drum, and were then covered with additional fibres in a second step, resulting in a patterned matrix. Fibre diameters were statistically different for all comparisons; displaying $265 \pm$ $97 \mathrm{~nm}$ on randomly oriented matrices, $354 \pm 156 \mathrm{~nm}$ on parallel oriented matrices and $148 \pm 43 \mathrm{~nm}$ on patterned matrices. Matrix morphologies are displayed in figure 3.

Mechanical properties were determined based on stressstrain measurements and reported in table 2. No significant differences among anisotropic substrates (micropatterned or unpatterned) were found. As might be expected, n-RO matrices possessed higher elongation at break values due to reassembly of the fibrous structure prior to fibre drawing and breaking. Similarly, Kim (2008) reported distinct mechanical properties of PCL electrospun membranes depending on fibre or stress orientation.

To our knowledge, few methods to pattern fibrous electrospun substrates have been documented. Importantly, this approach permits the combination of two well-known triggers of cell alignment that have usually been studied individually. Micropatterned structures have been incorporated on electrospun membranes of polyurethanes or PCL/gelatine blends using either femtosecond-pulse laser ablation (Lim et al 2011) or silicone moulds patterned with soft lithography (Dempsey et al 2010). Both methods resulted in the generation of sharp surface topographies of varying groove widths and depths of 20-500 $\mu \mathrm{m}$, depending on the method. Of note, our substrates display a continuous, wavy topography without clear boundaries. These characteristics are of particular importance as, firstly, this topography comes close to the grooved architecture of native muscle tissue; and, secondly, the fibrous matrices architecturally mimic the fibrous nature of the extracellular matrix, thereby providing an adequate environment for cell adhesion and orientation. 

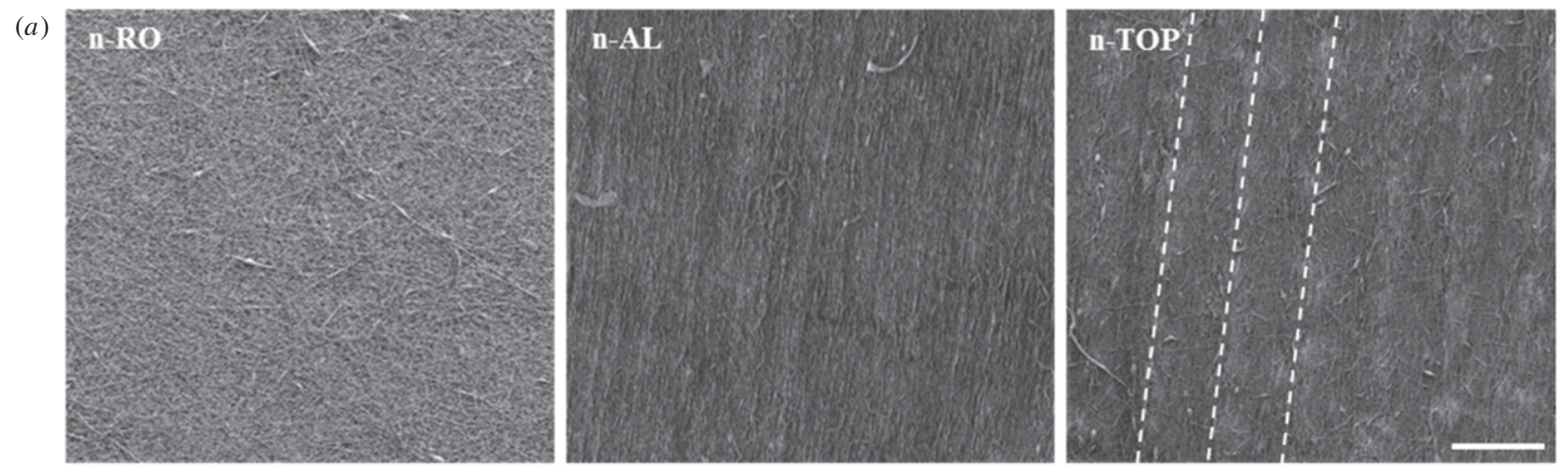

(b)
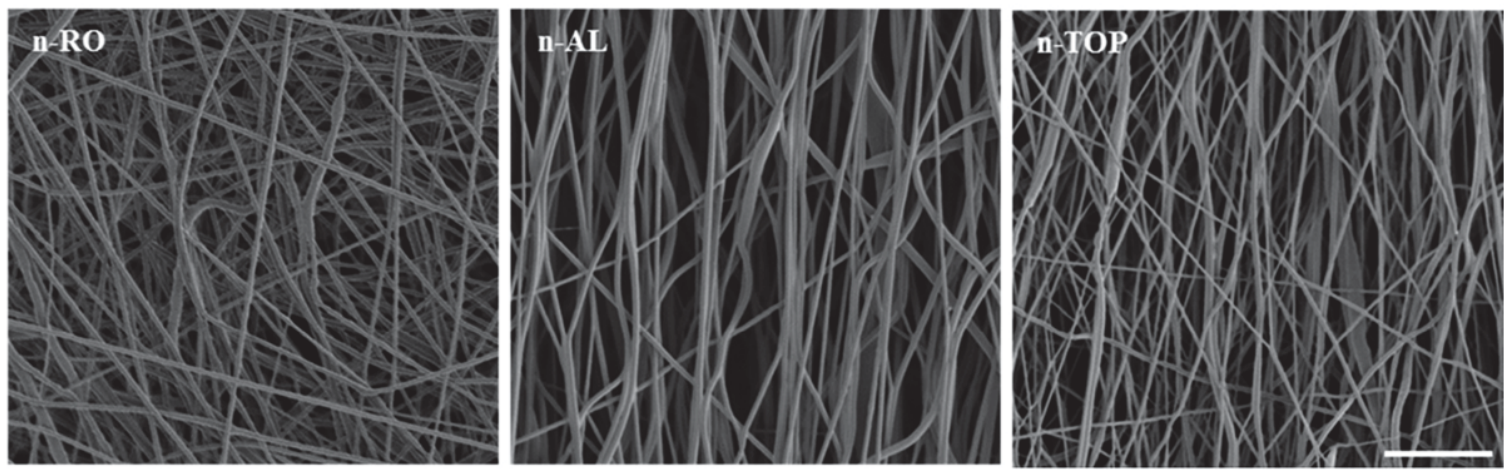

Figure 3. SEM images of randomly oriented (n-RO), parallel-oriented (n-AL) and patterned (n-TOP) electrospun matrices at low (a) and high $(b)$ magnifications. The patterned surface of the matrix is highlighted with dashed lines, showing grooves of the matrix. On n-TOP substrates, fibre orientation is less pronounced than on $\mathrm{n}$-AL substrates. Scale bars represent $100 \mu \mathrm{m}(a)$ and $5 \mu \mathrm{m}(b)$.

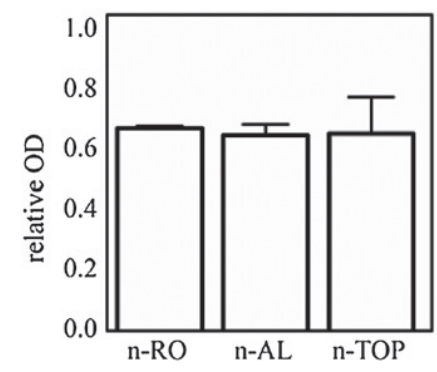

Figure 4. Cell viability was assessed with an MTT test. OD relative to TCPS was $0.66 \pm 0.01$. Cell viability did not differ among conditions.

\subsection{Cell viability and proliferation}

MTT analysis, LDH release and cell proliferation assays confirmed the presence of viable cells on the three distinct substrates. LDH corresponded to $6 \pm 1 \%$ of that for total lysed cells and was comparable to LDH release in standard TCPS cell cultures. An MTT test performed $24 \mathrm{~h}$ after seeding further confirmed cell viability on all substrates. Spectrophotometrical analysis revealed an optical density (OD) of $0.66 \pm 0.01$ relative to $\mathrm{C} 2 \mathrm{C} 12$ culture on TCPS (figure 4). No significant differences were found among conditions. The MTT test confirmed cytocompatibility of the designed substrates.

For myoblast fusion and myotube formation to occur, close proximity and orientation of cells is mandatory. Consequently, cell confluence on the culture substrate is of paramount importance for onset of differentiation. Cell number on the respective substrates was assessed at three time points in order to define the optimal time for addition

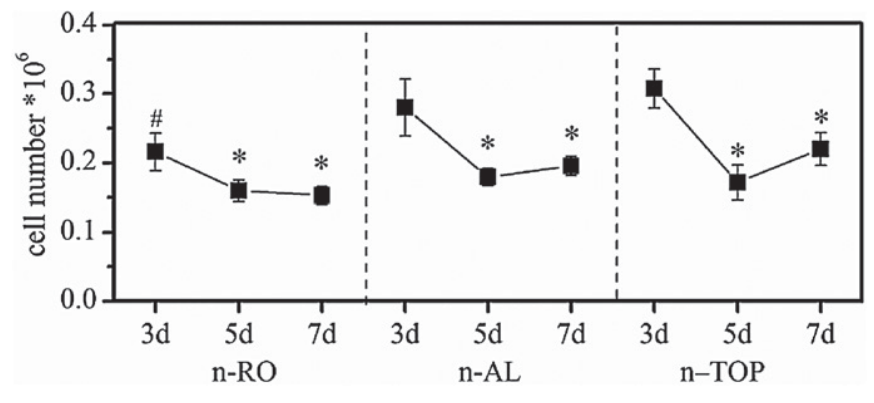

Figure 5. Cell number was assessed with CyQuant DNA quantification. Cell number was highest three days post seeding and significantly decreased with time. $\left({ }^{*} p<0.01\right.$ compared to day 3 with respective substrate; $\# p<0.01$ compared to day 3 with n-TOP substrates; $n=12$, power $\pi>0.98$ ).

of differentiation medium, based on maximal cell number and confluence. Following initial seeding with an aliquot of $0.12 \pm$ $0.02 \times 10^{6}$ cells, cell number reached a peak after three days of culture.

The growth phase was followed by a decrease in cell number over time (figure 5). Accordingly, myotube differentiation was initiated three days post seeding.

\subsection{Myoblast orientation}

The orientation parameter for myoblasts cultured on nRO fibres was $S=0.35 \pm 0.2$, indicating random cell orientation. Conversely, orientation parameters calculated for cells cultured on both anisotropic substrates were significantly higher, $0.65 \pm 0.1$ and $0.67 \pm 0.1$ for $n-A L$ or $n-T O P$, 

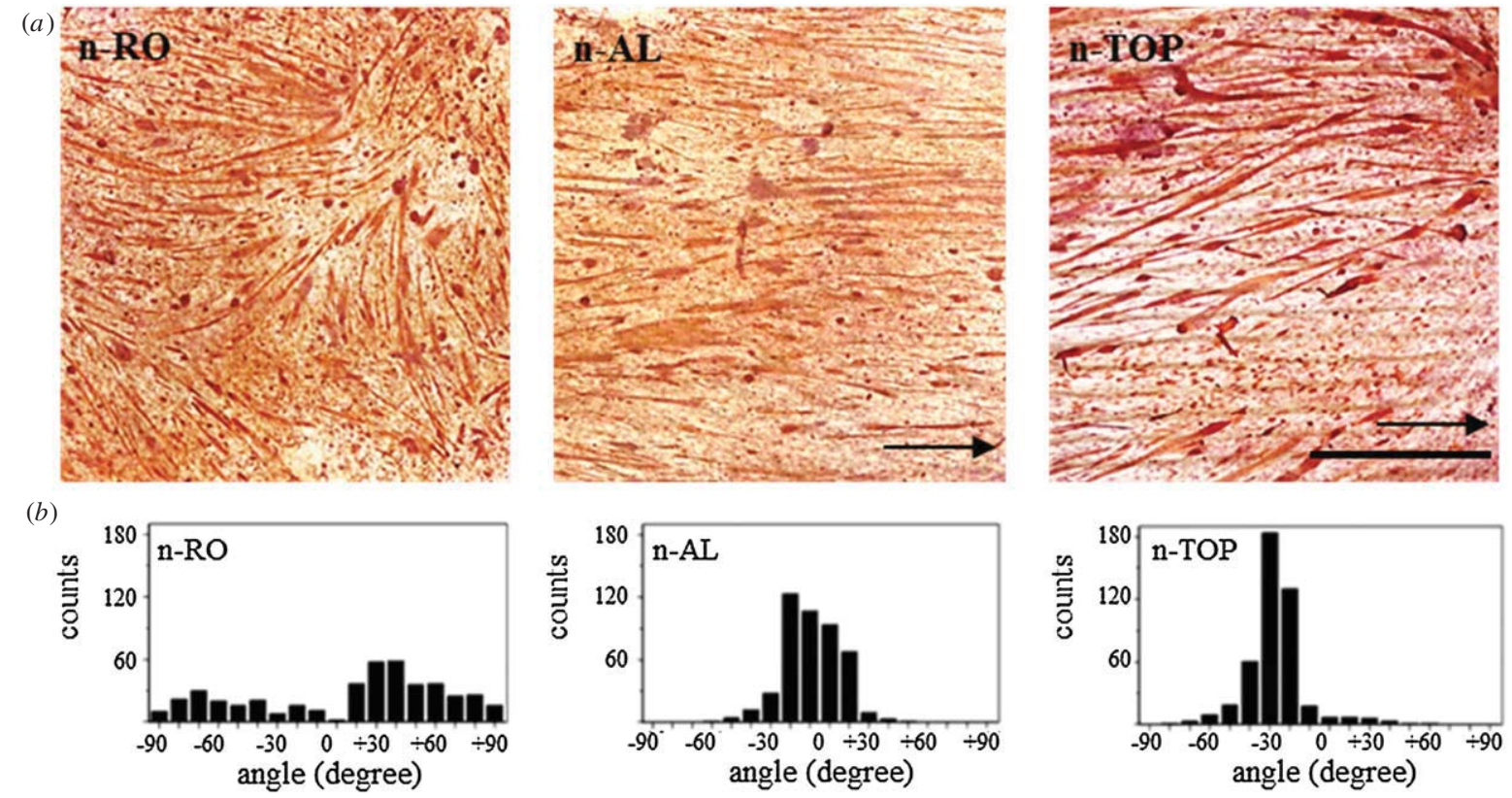

Figure 6. (a) Light microscopy images of desmin ${ }^{(+)}$stained myotubes on $\mathrm{n}-\mathrm{RO}, \mathrm{n}$-AL and n-TOP substrates. Arrows indicate fibre orientation. Scale bar represents $1 \mathrm{~mm}$. (b) Histogram of myotube angle relative to fibre orientation. Myotubes on n-RO substrates were randomly oriented, displaying a spiral-like pattern. On n-AL substrates, myotubes followed fibre alignment, whereas myotubes on patterned substrates were oriented at an angle of $21 \pm 5^{\circ}\left(\right.$ median of $\left.24^{\circ}\right)$ relative to fibre orientation.

respectively. These results are in accordance with previous reports, in which contact guidance has been reported to significantly impact upon myoblast spatial orientation (represented by a direct correlation of fibre orientation or topography with cell orientation). Conventional approaches for myoblast orientation have utilized lithography to create micropatterns of varying geometry on flat substrate such as PDMS. In these conditions, periodic grooves/ridges have been demonstrated to influence myoblast orientation and myotube formation. Lam et al (2006) reported increased myoblast alignment and myotube formation on continuous waves of $6 \mu \mathrm{m}$ periodicity, whereas Charest et al (2007) found groove widths of 5, 10 or $25 \mu \mathrm{m}$ adequate. Another study (Altomare et al 2010) found that structures of 25-50 $\mu \mathrm{m}$ induced myoblast alignment. In addition, sub-micrometer topographies displaying groove sizes of $450-900 \mathrm{~nm}$ were found to promote myoblast alignment and fusion into longer, more regularly shaped myotubes in comparison with flat substrates. Similar to reports of substrates with grooves of various widths and depths, electrospun fibres of various polymers in the nanometre or micrometre range were reported to induce spatial myoblast orientation and fusion into multinucleated myotubes (Choi et al 2008, Riboldi et al 2008, Aviss et al 2010). Various studies led to the hypothesis that a wide range of fibre diameter ( $300 \mathrm{~nm}$ to $12 \mu \mathrm{m}$ ) can induce myoblast orientation. Previous studies of our group (Guex et al 2012), however, have demonstrated that spiral-like orientation patterns of cells on parallel-oriented nanoscaled fibres were comparable to commonly observed patterns in confluent TCPS cultures (Palumberi et al 2006). Hypothetically, contact guidance is restricted at a certain diameter threshold. The significantly smaller fibres of n-TOP substrates did not lead to a spiral-like pattern, suggesting that the micropattern added additional cues for parallel orientation of myoblasts.

\subsection{Myotube differentiation and orientation}

Under partial serum deprivation for ten days, myoblasts fused into multinucleated myotubes on all substrates. Light microscopy of desmin ${ }^{(+)}$stained substrates revealed elongated, multinucleated, unbranched myotubes (figure 6(a)). On randomly oriented fibres, myotubes assembled into circle or spiral-like patterns in a comparable manner to the orientation of non-differentiated myoblasts on the same substrate. On parallel-oriented substrates without surface pattern, elongated myotubes assembled according to fibre orientation into parallel patterns. Orientation parameters were $0.24 \pm 0.15$ and $0.87 \pm$ 0.01 on $\mathrm{n}-\mathrm{RO}$ and $\mathrm{n}-\mathrm{AL}$, respectively.

Interestingly, micropatterned substrates induced divergent myotube orientation. $70 \%$ of myotubes assembled at an angle of $21 \pm 5^{\circ}$ (statistical median of $24^{\circ}$ ) relative to the periodic pattern and not according to fibre direction, as reported for parallel-oriented fibres without an additional surface topography (figure 6). A comparative study of grooved PDMS substrates and PDMS posts by Gingras et al (2009) reported on an unexpected alignment of myotubes with a mean angle of $25 \pm 5^{\circ}$ relative to the symmetry of the posts. The findings were explained by a computational geometric model of the initially migrating myoblasts. Alignment along the patterns at a certain angle was suggested to provide maximal adhesion surface area. Conversely, myoblast orientation in our studies was parallel along the grooves whereas only myotube orientation displayed a different pattern.

Taken together, these results provide evidence that contact guidance of myotubes on the fibrous topography is different 
Table 3. Myotube width and length.

\begin{tabular}{llll}
\hline Matrix & $\begin{array}{l}\text { Myotube width } \\
(\mu \mathrm{m})\end{array}$ & $\begin{array}{l}\text { Myotube length } \\
(\mu \mathrm{m})\end{array}$ & $\begin{array}{l}\text { Area coverage } \\
(\%)\end{array}$ \\
\hline $\mathrm{n}-\mathrm{RO}$ & $11 \pm 5$ & $255 \pm 143$ & $8.4 \pm 7$ \\
$\mathrm{n}-\mathrm{AL}$ & $14 \pm 6^{\mathrm{a}}$ & $365 \pm 212^{\mathrm{a}}$ & $24.7 \pm 7$ \\
$\mathrm{n}-\mathrm{TOP}$ & $15 \pm 7^{\mathrm{a}}$ & $436 \pm 245^{\mathrm{b}}$ & $14.5 \pm 14$ \\
\hline
\end{tabular}

${ }^{\text {a }} p<0.05$ compared to $\mathrm{n}-\mathrm{RO}(n=300$, power $\pi=1)$.

${ }^{\mathrm{b}} p<0.05$ compared to $\mathrm{n}-\mathrm{RO}$ and $\mathrm{n}-\mathrm{AL}(n=300$, power $\pi=$ $0.97)$.

than myoblast guidance. In contrast, myotube orientation on two dimensional, non-fibrous patterns was reported as parallel to the grooves (Lam et al 2006, Altomare et al 2010, Wang et al 2010). Our observations suggest that a lateral fusion of the myoblast is favoured by the underlying fibrous matrix.

Furthermore, Zhao et al (2009) initiated a double seeding strategy, where additional myoblasts were seeded on differentiated myotubes cultured on micropatterned PDMS substrates. The additional layer of myotubes differentiated in a much quicker time-frame and resulted in an increased myotube width in comparison to the first layer. These results support the importance of creating either a surface topography that closely mimics the native substrate of extracellular matrix proteins and myotubes during myogenesis or the incorporation of multiple seeding steps. In our study, myotube differentiation was significantly enhanced on both anisotropic matrices compared to $\mathrm{n}-\mathrm{RO}$ matrices. Percentage coverage of myotubes were 8.4 $\pm 7 \%$ on $\mathrm{n}-\mathrm{RO}, 24.7 \pm 7$ on $\mathrm{n}$-AL and $14.5 \pm 14$ on $\mathrm{n}$-TOP matrices. On n-RO matrices, myotubes were also observed to be significantly shorter than on substrates of aligned fibres or micropatterned substrates of aligned fibres. Myotubes on n-TOP substrates displayed a mean length of $436 \pm$ $245 \mu \mathrm{m}$ that was significantly longer than myotubes on $\mathrm{n}-\mathrm{AL}$ substrates $(365 \pm 212 \mu \mathrm{m} ; p<0.05)$. Myotube width, however, was comparable on both anisotropically structured substrates, yet significantly larger than on n-RO substrates. Values are presented in table 3 . The standard interpretation of the experimental results based on the $p$ values was completed by a retrospective power calculation. The large sample size allowed us to increase the precision of the mean of measured myotube length and width and resulted in a high power as presented in table 3 .

Although the difference in myotube length was relatively small, we can conclude that an imprinted micropattern on parallel-oriented fibres has a pronounced effect on myotube spatial orientation and induced the formation of longer myotubes. Myotube width and density were, however, comparable to substrates without micropattern. Our results highlight the importance of contact guidance in inducing myotube fusion and enhancing muscle tissue generation. The simple approach of generating dual-level topographical matrices by electrospinning onto topographically structured collectors provides an interesting approach for the design of novel fibrous substrates. By varying the engraved topography on the drum, scaffolds displaying various groove widths and depths can easily be produced. To gain further insight into the spatial orientation of myotubes on architecturally structured scaffolds and their differentiation into mature myotubes, we suggest a thorough study on groove width and depth in order to optimize the culture substrate for further studies.

\section{Conclusion}

We report a one-step method to produce matrices of aligned, electrospun fibres with an additional micron-scaled surface topography. By using a patterned and rotating counter electrode, a mirrored matrix of periodic grooves was obtained. We demonstrate that the unique combination of nanofibrous matrices and micron-scaled periodic grooves creates a substrate for muscle tissue engineering that closely resembles native myogenesis. The periodic grooves induced the formation of long myotubes and the orientation of myotubes with a $24^{\circ}$ deviation with respect to fibre orientation. Further studies on distinct surface properties are needed to provide insight into myotube formation on architecturally distinct surfaces and would contribute to the amelioration of substrate design for muscle tissue engineering. Micropatterning of electrospun substrates provides an additional innovative method for scaffold design and is worth being pursued in future investigations.

\section{Acknowledgments}

The authors acknowledge financial support from the Swiss Science Foundation (grant 122334) and the Inselspital Bern. The authors would also like to thank Dr Patrick Rupper, Empa, St Gallen for assistance during SEM imaging, Pierluigi Barbadoro, Empa St Gallen for assistance during stress-strain measurement, Brigitta Gahl, Inselspital Bern for statistical analysis, Céline Fouassier, Inselspital Bern, for general lab management and Dr Sarah Longnus, Inselspital Bern, for English proof reading.

\section{References}

Altomare L, Gadegaard N, Visai L, Tanzi M C and Farè S 2010 Biodegradable microgrooved polymeric surfaces obtained by photolithography for skeletal muscle cell orientation and myotube development Acta Biomater. 6 1948-57

Aviss K J, Gough J E and Downes S 2010 Aligned electrospun polymer fibers for skeletal muscle regeneration Eur. Cells Mater. 19 193-204

Biela S A, Su Y, Spatz J P and Kemkemer R 2009 Different sensitivity of human endothelial cells, smooth muscle cells and fibroblasts to topography in the nano-micro range Acta Biomater. 5 2460-6

Charest J L, Garcia A J and King W P 2007 Myoblast alignment and differentiation on cell culture substrates with microscale topography and model chemistries Biomaterials 28 2202-10

Choi J S, Lee S J, Christ G J, Atala A and Yoo J J 2008 The influence of electrospun aligned poly(epsilon-caprolactone)/ collagen nanofiber meshes on the formation of self-aligned skeletal muscle myotubes Biomaterials 29 2899-906

Dempsey D K, Schwartz C J, Ward R S, Iyer A V, Parakka J P and Cosgriff-Hernandez E M 2010 Micropatterning of electrospun polyurethane fibers through control of surface topography Macromol. Mater. Eng. 295 990-4 
Duxson M J, Usson Y and Harris A J 1989 The origin of secondary myotubes in mammalian skeletal muscles: ultrastructural studies Development 107 743-50

Engler A J, Griffin M A, Sen S, Bonnetnann C G, Sweeney H L and Discher D E 2004 Myotubes differentiate optimally on substrates with tissue-like stiffness: pathological implications for soft or stiff microenvironments J. Cell Biol. $166877-87$

Erickson C A and Nuccitelli R 1984 Embryonic fibroblast motility and orientation can be influenced by physiological electric-fields J. Cell Biol. 98 296-307

Gingras J, Rioux R M, Cuvelier D, Geisse N A, Lichtman J W, Whitesides G M, Mahadevan L and Sanes J R 2009 Controlling the orientation and synaptic differentiation of myotubes with micropatterned substrates Biophys. J. 97 2771-9

Guex A G, Kocher F M, Fortunato G, Korner E, Hegemann D, Carrel T P, Tevaearai H T and Giraud M N 2012 Fine-tuning of substrate architecture and surface chemistry promotes muscle tissue development Acta Biomater. 8 1481-9

Hauschka S 1994 Myology ed A G Engel and C Franzini-Armstrong (New York: McGraw-Hill) pp 3-73 (The Embryonic Origin of Muscle)

Kim G H 2008 Electrospun PCL nanofibers with anisotropic mechanical properties as a biomedical scaffold Biomed. Mater. 3025010

Koning M, Harmsen M C, van Luyn M J A and Werker P M N 2009 Current opportunities and challenges in skeletal muscle tissue engineering J. Tissue Eng. Regen. Med. 3 407-15

Lam M T, Sim S, Zhu X and Takayama S 2006 The effect of continuous wavy micropatterns on silicone substrates on the alignment of skeletal muscle myoblasts and myotubes Biomaterials 27 4340-7

Lenth R 2006-2009 Java applets for power and sample size http://www.stat.uiowa.edu/rrlenth/Power

Lim Y C, Johnson J, Fei Z, Wu Y, Farson D F, Lannutti J J, Choi H W and Lee L J 2011 Micropatterning and characterization of electrospun poly(epsilon-caprolactone)/ gelatin nanofiber tissue scaffolds by femtosecond laser ablation for tissue engineering applications Biotechnol. Bioeng. 108 116-26

Moghe A K, Hufenus R, Hudson S M and Gupta B S 2009 Effect of the addition of a fugitive salt on electrospinnability of poly(epsilon-caprolactone) Polymer $503311-8$
Ontell M 1977 Neonatal muscle: an electron microscopic study Anat. Rec. 189 669-90

Palumberi V, Barbero A, Grote M, Martin I and Wagner B 2006 Spatio-angular modeling for the formation of oriented patches in chondrocyte cultures Coloque Numérique Suisse (Lausanne, Switzerland)

Riboldi S A, Sadr N, Pigini L, Neuenschwander P, Simonet M, Mognol P, Sarnpaolesi M, Cossu G and Mantero S 2008 Skeletal myogenesis on highly orientated microfibrous polyesterurethane scaffolds J. Biomed. Mater. Res. A 84 1094-101

Riboldi S A, Sampaolesi M, Neuenschwander P, Cossu G and Mantero S 2005 Electrospun degradable polyesterurethane membranes: potential scaffolds for skeletal muscle tissue engineering Biomaterials 26 4606-15

Ricotti L, Polini A, Genchi G G, Ciofani G, Iandolo D, Vazao H, Mattoli V, Ferreira L, Menciassi A and Pisignano D 2012 Proliferation and skeletal myotube formation capability of $\mathrm{C} 2 \mathrm{C} 12$ and $\mathrm{H} 9 \mathrm{c} 2$ cells on isotropic and anisotropic electrospun nanofibrous PHB scaffolds Biomed. Mater. 7035010

Rossi C A, Pozzobon M and De Coppi P 2010 Advances in musculoskeletal tissue engineering moving towards therapy Organogenesis 6 167-72

Shimizu K, Fujita H and Nagamori E 2009 Alignment of skeletal muscle myoblasts and myotubes using linear micropatterned surfaces ground with abrasives Biotechnol. Bioeng. 103 631-8

Wakelam M J 1985 The fusion of myoblasts Biochem. J. $2281-12$

Wang P-Y, Yu H-T and Tsai W-B 2010 Modulation of alignment and differentiation of skeletal myoblasts by submicron ridges/grooves surface structure Biotechnol. Bioeng. $106285-94$

Wigmore P M and Dunglison G F 1998 The generation of fiber diversity during myogenesis Int. J. Dev. Biol. 42 117-25

Zhang M and McLennan I S 1995 During secondary myotube formation, primary myotubes preferentially absorb new nuclei at their ends Dev. Dyn. 204 168-77

Zhao Y, Zeng H S, Nam J and Agarwal S 2009 Fabrication of skeletal muscle constructs by topographic activation of cell alignment Biotechnol. Bioeng. 102 624-31 